\title{
A Novel Backoff Algorithm with QoS Support in Heterogeneous Wireless Networks
}

\author{
A. Rajesh, Member, IACSIT and R. Nakkeeran
}

\begin{abstract}
The main objective of the future networks is to provide users with always best connectivity through available different access networks. Wireless convergence has emerged as a multidimensional concept that combines networks, applications, user interfaces and management, which aimed at supporting voice, data and multimedia over IP-based networks. In this paper, we model the end-to-end quality of service (QoS) provisioning mechanisms in the wireless local area network (WLAN) and worldwide interoperability for microwave access networks (WiMAX) interworking network. For the end to end QoS guaranteed service, we make changes to multiple access protocol. The media access control (MAC) analysis using hybrid protocol based on virtual slot multiple access (VSMA) with a novel backoff mechanism is extended to the heterogeneous networks and its throughput and delay characteristics are analyzed. By the simulation results, our proposed protocol can provide the low end to end delay and improved throughput for different traffics, even though the traffic load in the network increases. Especially, the end-to-end delay of the IP based voice is smaller when the QoS parameter mappings are applied to the interworking network.
\end{abstract}

Index Terms-Backoff mechanism, medium access control, WiMAX, WLAN.

\section{INTRODUCTION}

The advent of ubiquitous computing and the growth of portable computing devices have raised the importance of mobile and wireless networking. With the rapid growth of the Internet and interactive facilities of multimedia-based services, the current wireless technology is flourishing in every aspect of human life ranging from home, education, medicine, e-commerce and m-commerce. Today, almost every network technology is prepared or even explicitly designed to transport IP-packets. To meet the ever increasing consumer demands the mobile networks have evolved from first generation $(1 \mathrm{G})$ to fourth generation $(4 \mathrm{G})$ [1].

In order to ensure consistent user experience across heterogeneous wireless networks, the main challenge is to provide end-to-end QoS and to optimize transmission of all data, voice, and video applications to and among end users. The performance needs of applications are characterized by four key parameters: bandwidth, packet loss, delay, and jitter (i.e., delay variation), which determine the QoS.

Overall, the needs of the distinct applications are different with respect to QoS. For example, some popular data applications (such as e-mail and Web access) require low to

Manuscript received February 15, 2012; revised April 25, 2012.

The authors are with the Department of Electronics Engineering, School of Engineering and Technology, Pondicherry Central University, Puducherry, INDIA (e-mail: rajeshatnet@gmail.com; rnakeeran@ pec.edu). medium bandwidth and are quite relaxed as far as the delay and jitter are concerned. On the other hand, Video-on-Demand (VoD) flows have relaxed requirements on delay, but they do need high bandwidth and cannot tolerate much packet loss or jitter. Voice over IP (VoIP), while tolerating some packet loss, needs much lower bandwidth than $\mathrm{VoD}$, but it can tolerate neither long delay nor jitter. Delay becomes perceptible to the user somewhere between 150 and $400 \mathrm{~ms}$, and some network operators specify maximum delay in the range of 250-300 ms. Jitter should stay under $50 \mathrm{~ms}$ [2].

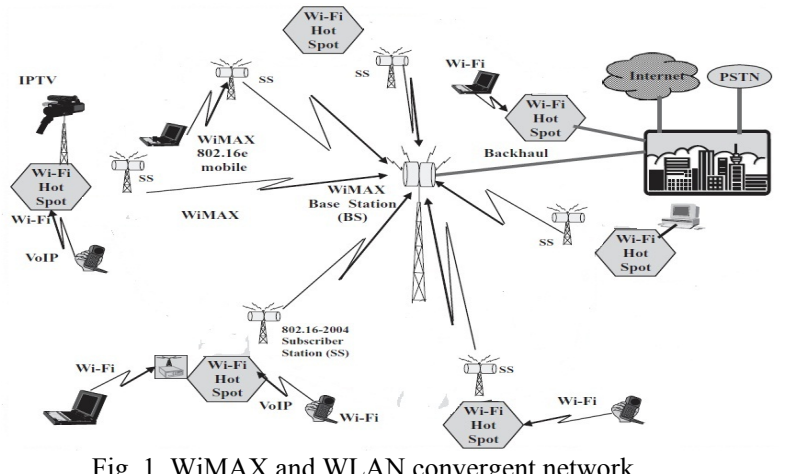

During recent years, IEEE802.11 Wireless Local Area Networks (WLAN) have been deployed widely and 802.11 access points (APs) are able to cover areas of a few thousand square meters, making them suitable for enterprise networks and public hot spot scenarios such as airports and hotels [3]. Recently, WiMAX using the IEEE802.16e standard received a great deal of attention because of the high rate of data support, its intrinsic QoS and mobility capabilities and a much wider area of coverage enabling ubiquitous connectivity [4]. An interworking between these technologies has been considered as a viable option for the realization of the $4 \mathrm{G}$ scenario as shown in Fig. 1. However, this interoperation raises several challenges especially when seamless session continuity is required for, for example, media calls such as VoIP or video telephony. Since the WiMAX and the WLAN have different protocol architectures and QoS support mechanisms, protocol adaptation would be required for their interworking [5]-[6]. For example, with a layer 2 approach, adaptation would be required in the MAC layer for the WiMAX base station (BS) and WLAN nodes [7].

In this paper, we propose two solutions for a WLAN/WiMAX interconnection by taking into account two main goals: Traffic priority and implementation issues. The first solution is more based on the concept of maintaining a certain end to end QoS level independently from the wireless technologies used to link them. In that sense, the primitives of 
the MAC layer of the two protocol stacks have been considered in order to assure the requested QoS level for a certain connection. The second solution is more dedicated to the reduction of the implementation complexity and limiting the protocol adaptation between the two stacks. The main objective of the proposed heterogeneous networks is to provide users with always best connectivity through available different access networks even the user is on move. The second solution utilizes the concept of virtual slot multiple access (VSMA) with a novel backoff mechanism.

The VSMA is based on the concept of hybridizing carrier sense multiple accesses with collision avoidance (CSMA/CA) and time division multiple access (TDMA) schemes [8]. The VSMA uses idle slot reuse scheme. In CSMA/CA, a binary exponential backoff algorithm is being employed and with a fixed contention window $(\mathrm{CW})$, the channel is not used efficiently and effectively. Also to meet the increasing user demand for multimedia applications, an enhanced backoff mechanism has to be incorporated to maintain the throughput and to reduce the delay of the network [9]-[10]. In this paper, we propose a dynamic $\mathrm{CW}$ based access mechanism and our proposed algorithm reduces the collision rate and wasted idle time which in turn increases the MAC throughput and can provide multimedia application even under worst channel conditions.

The rest of the paper is organized as follows. Section II describes the proposed heterogeneous network and a hybrid access scheme at the MAC layer. Also we propose a novel backoff scheme in Section III. In Section IV, we present the performance evaluation and discuss about the results and finally we conclude the paper in Section V.

\section{Proposed Heterogeneous System}

One vital component for the provision of seamless multimedia session continuity is QoS consistency across the WLAN and Mobile WiMAX networks. This is vital because without QoS consistency the multimedia sessions will experience different QoS levels in both network domains, and thus seamless continuity will not be achievable. It is unfortunate, however, that the WLAN and Mobile WiMAX specifications were based on different sets of requirements, and ended up supporting different sets of QoS features. Consequently, QoS consistency turns out to be a very challenging issue. When we target multimedia session continuity across WLAN and Mobile WiMAX networks, we considered these deficiencies into consideration. The discussion is based on the assumption that the WLAN MAC layer complies with IEEE 802.11 plus the amendments of IEEE 802.11e, and the physical layer complies with IEEE $802.11 \mathrm{~g}$ while mobile WiMAX is based on IEEE 802.16e the mobile version of IEEE 802.16d. The simulated network architecture is shown in Fig. 2.

The PHY layer is divided in two significant parts: The first including 10-66 GHz frequencies and the second regarding the $2-11 \mathrm{GHz}$ band. For the $10-66 \mathrm{GHz}$, the line-of-sight (LOS) propagation is needed due to the high working frequencies while the $2-11 \mathrm{GHz}$ band is driven by the need for non line-of-sight (NLOS) operation [11]. The access protocol can be either TDMA or frequency division multiple access (FDMA), with both time division duplex (TDD) and frequency division duplex (FDD). The physical parameters considered in our proposed system uses a channel bandwidth of 20, 25, or $28 \mathrm{MHz}$, with QPSK, 16-QAM, and 64-QAM modulations and frame durations of $0.5,1$, and $2 \mathrm{~ms}$.

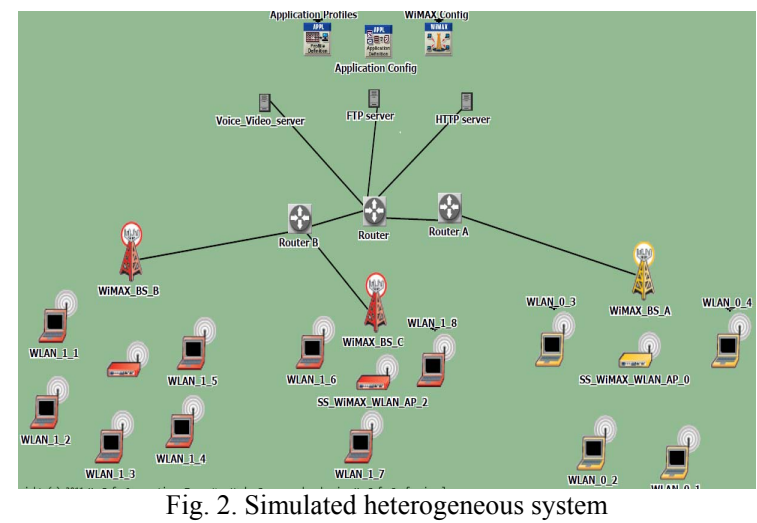

\section{A. Medium Access Control (MAC) Layer}

In the proposed heterogeneous model, the MAC layer at the base station is responsible for allocating bandwidth to all users, in both the uplink and the downlink. The only time the mobile station (MS) has some control over bandwidth allocation is when it has multiple sessions or connections with the base station (BS). Depending on the particular QoS and traffic parameters associated with a service, one or more of these mechanisms may be used by the MS. The BS allocates dedicated or shared resources periodically to each MS, which it can use to request bandwidth.

The most important feature of the hybrid protocol is the access to the channel that is based on priorities of the packets queued in stations. If the packets are found real time then the MS is allowed to access the channel using TDMA mechanism. If they found non-real time then it is allowed for CSMA/CA mechanism. The CSMA/CA is used instead of TDMA to improve channel utilization. This also enhances a huge amount of bursty traffic to pass through the empty slot. The Fig. 3 describes the procedure of VSMA in detail using flowchart.

The SNR is an important parameter and it depends on data packets computing. The value of the parameter will indicate the MAC to classify the kind of traffic to send according to medium quality. If $(\mathrm{SNR})<(\mathrm{SNR})_{\mathrm{Th}}$, then the station differs emission if it has QoS traffic, otherwise it emits the best effort traffic. If $(\mathrm{SNR}) \geq(\mathrm{SNR})_{\mathrm{Th}}$, then station transmits best effort or QoS traffic.

\section{Proposed BACKOFF ALGORITHM}

In literature a standard algorithm like exponential increase exponential decrease has been addressed for choosing the optimum contention window but the algorithm did not account for individual priority. Hence we mainly focus on choosing the contention window $(\mathrm{CW})$ adaptively based on priority for three different conditions. The amount of increase or decrease of the $\mathrm{CW}$ when a station experiencing a collision, overhearing a collision and for a success for different priority is discussed in detail as follows: 


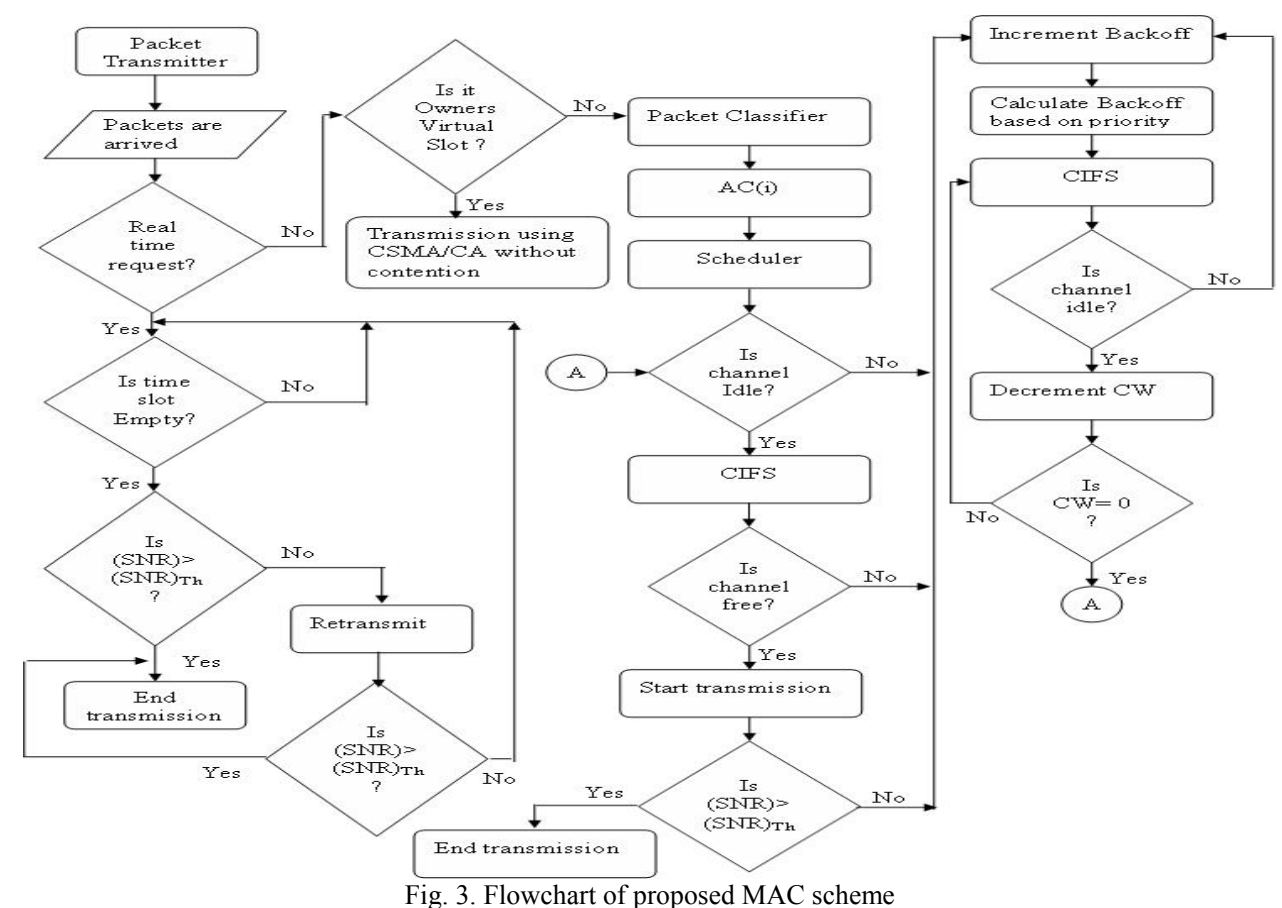

TABLE I:CW SELECTION MECHANISM BASED ON PRIORITY OF SERVICE

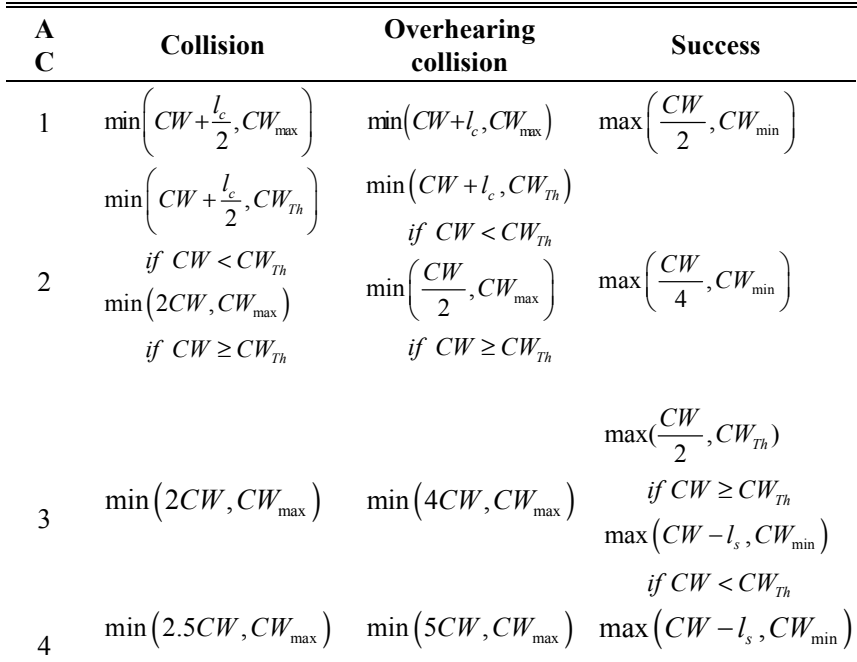

The basic idea behind our method is that, prioritized access to the WLAN medium is controlled through different $\mathrm{CW}$ periods. The $\mathrm{CW}$ generation function has been set to 0 to $\left(2^{2+\mathrm{i}+\mathrm{k}}-1\right)$ for high priority stations and $\left(2^{2+\mathrm{i}+\mathrm{k}}\right)$ to $\left(2^{2+\mathrm{i}+\mathrm{k}}-1\right)$ for low priority stations respectively. A brief idea about the selection of CW for different priorities is shown in Table I.

The access category $1(\mathrm{AC} 1)$ is considered as very high priority services and so on, and if a station notices that its packet transmission has failed due to packet collision, the contention window size of the station will be increased linearly and a random backoff time (BT) will be chosen. The stations in the deferring state, whenever detects the start of a new busy period, which indicates either a collision or a packet transmission in the medium, the stations will increase their contention window size linearly. If a station has finished a successful packet transmission, then the contention window size of other stations will be reduced multiplicatively until to their initial (minimum) contention window size. In all these cases the $\mathrm{BT}=$ rand $(0, \mathrm{CW}-1) \times$ Slot-Time and rand indicates a number randomly drawn from distribution between 0 and $\mathrm{CW}$.

\section{RESUlts AND Discussion}

The simulation environment is implemented using OPNET 14.5. The OPNET WiMAX Specialized Model supports the IEEE 802.11n and IEEE 802.16e standards. It can be used to evaluate custom scheduling algorithms for WiMAX/WLAN base and subscriber stations for different MAC and PHY layer profiles. The simulation parameters are shown in Table II. We tested IP based voice, video and data traffic (HTTP) in our proposed VSMA based heterogeneous wireless network. Each corresponding node is running voice, video and data application with its corresponding access point.

TABLE II: SIMULATION PARAMETERS AT MAC LAYER

\begin{tabular}{ll}
\hline \hline Parameter & Details \\
\hline Protocol & VSMA \\
Number of stations & 12 \\
Traffic & IP based Voice, Video, \\
Physical channel rate & $50 \mathrm{Mb} / \mathrm{s}$ \\
MAC frame size & $50-1500$ bytes \\
CIFS & $35.84 \mu_{\mathrm{s}}$ \\
PRS0, PRS1 & $35.84 \mu_{\mathrm{s}, 35.84} \mu_{\mathrm{s}}$ \\
Idle Slot time & $35.84 \mu_{\mathrm{s}}$ \\
RIFS & $26.0 \mu \mathrm{s}$ \\
Frame duration & $17 \mathrm{~ms}$ \\
Symbol duration & $71 \mu \mathrm{s}$ \\
Platform & Opnet 14.5 \\
\hline \hline
\end{tabular}




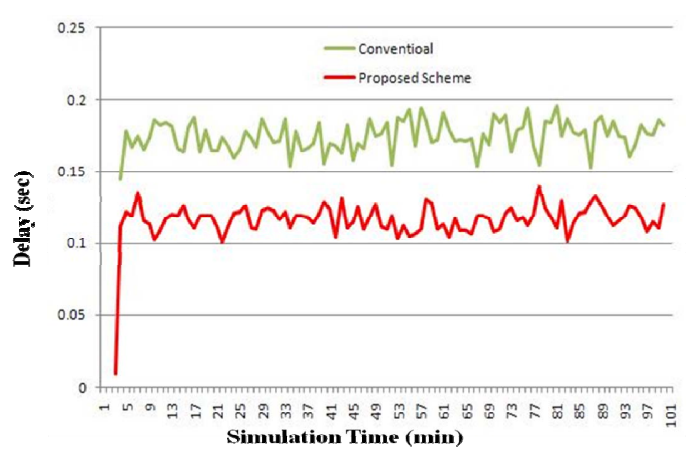

Fig. 4. Access delay of IP based voice traffic

We have simulated two scenarios namely the heterogeneous system with conventional MAC protocol and the system with our proposed VSMA based MAC mechanism. At each node in the heterogeneous network, differentiated QoS service are provided to treat four classes of traffic. In our proposed case, all four traffic classes were fed into the MAC layer from higher layer, which are corresponding to $\mathrm{AC}(1), \mathrm{AC}(2), \mathrm{AC}(3)$ and $\mathrm{AC}(4)$ respectively to check how efficient the new protocol is to provide service differentiation required for real time application.

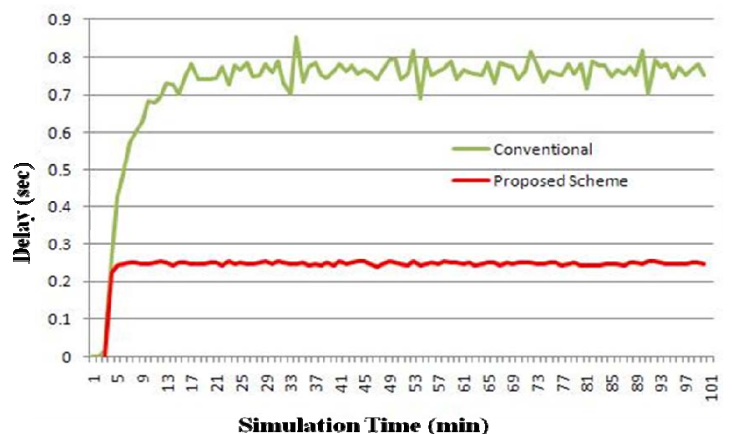

Fig. 5. Access delay of HD video traffic

The video traffic namely MPEG-4 is used for isochronous traffic and a HTTP traffic model for best effort traffic. Few nodes in the network are configured to support IP based voice traffic. HTTP traffic can increase the data rate up to channel capacity by TCP congestion control mechanism so that stations having HTTP traffic continuously try to access to the other station's virtual slot. If the stations do not use their virtual slots, they access the channel using only by TDMA protocol.

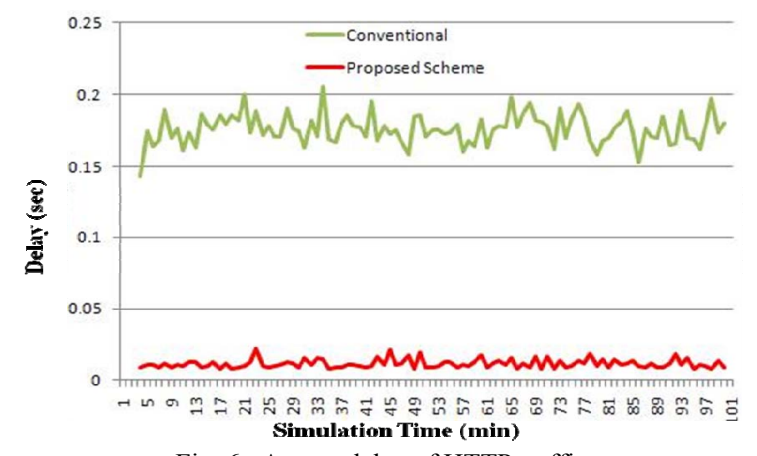

Fig. 6. Access delay of HTTP traffic

Fig. 4 shows the delay performance of TDMA and VSMA in IP based voice traffic flows. It shows the performance enhancement of the VSMA in terms of end to end delay, since the stations can transmit IP data packets through other stations' idle virtual slots. The delay curves shows less delay variation due to nature of delay sensitive voice traffic. From these reason, the VSMA supports the minimum delay requirement as the number of voice traffic in the network increases. Fig. 5 shows the delay performance of HD video traffic through the heterogeneous network. The proposed MAC mechanism shows an improvement of 50\% less delay in case of video traffic due to varying contention window mechanism.

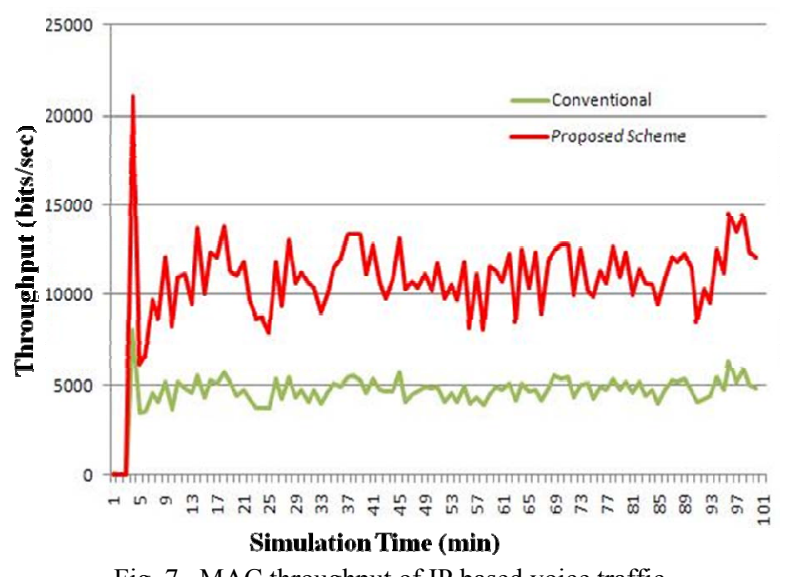

Fig. 7. MAC throughput of IP based voice traffic

Fig. 6 shows the delay performance for HTTP traffic. The end to end delay is poorer when the channel is unavailable for HTTP traffic as the slots are allocated for voice and video traffic. Thus, it increases message delay of HTTP traffic dramatically. As a result, the performance of TDMA with HTTP traffic is worst. However, VSMA mitigates this performance degradation considerably by allocating virtual slots and varying the contention window.

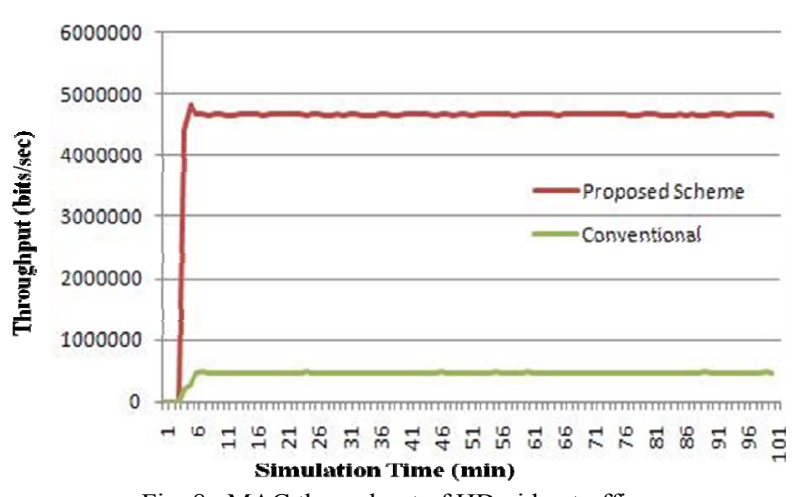

Fig. 8. MAC throughput of HD video traffic

Fig. 7 and Fig. 8 shows the performance enhancement of the proposed scheme in terms of the system throughput. As IP based voice and video flows through the channel increases, other stations can get a chance to transmit data packet through station's virtual slots. As a result, VSMA achieves higher total system throughput than TDMA. But there are too many variable Bit Rate (VBR) sources in the network so it causes a degradation of total system throughput of both of schemes. On the other hand, the portion of HTTP traffic to be smaller in the super frame. Fig. 8 shows very high throughput improvement for HD video traffic due to the nature of traffic and availability of other stations virtual time slots. 


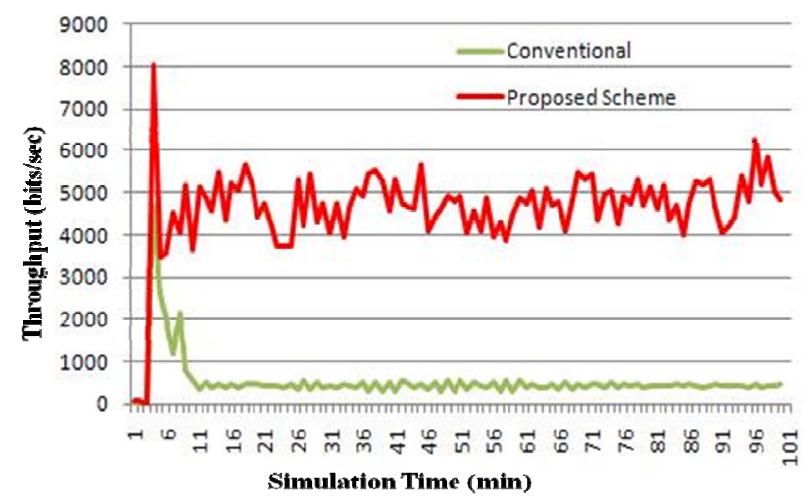

Fig. 9. MAC throughput of HTTP traffic

Fig. 9 shows that increase in the number of HTTP flows VSMA can have performance enhancement in terms of total system throughput. It is observed that the throughput improvement is much better when the HTTP is mixed with HD video since the characteristic of video traffic generates idle timeslots. And it can be used by other HTTP users. In conclusion, VSMA scheme makes the stations to effectively use the wireless channel resource by employing the virtual slot concept.

\section{CONCLUSION}

Heterogeneous networks are becoming more popular in delivering broadband services. The deployment of the proposed architecture allows users to switch seamlessly between these two types of network and provide several advantages to both users and service providers. By offering integrated WLAN/WiMAX services with QoS support, users would benefit from enhanced performance and high data rate of such a combined service. The hybrid protocol using VSMA has been investigated in accordance with the proposed backoff algorithm and the simulation results conclude that the proposed hybrid protocol shows an increase in throughput and decrease in delay when compared with the conventional scheme. The proposed integrated system is expected to serve as an excellent alternative to LTE (Long Term Evolution) systems in delivering broadband services to "last mile" and "last inch" connectivity.

\section{REFERENCES}

[1] A. K. Salkintzis, C. Fors, and R. Pazhyannur, "WLAN-GPRS integration for next-generation mobile data networks," IEEE Wireless Communications, vol. 9, no. 5, pp. 112-124, 2002.

[2] V. K. Varma, S. Ramesh, K. D. Wong, M. Barton, G. Hayward, and J. A. Friedhoffer, "Mobility management in integrated UMTS/WLAN networks," in Proc. of the IEEE International Conference on Communications, pp. 1048-1053, 2003.

[3] "Wireless LAN medium access control (MAC) and physical layer (PHY) specifications-amendment 8: Medium access control (MAC) quality of service enhancements," IEEE Std. 802.11e-2005.

[4] K. Yang, S. Ou, H. Chen, and J. He, "A Multihop Peer Communication Protocol with Fairness Guarantee for IEEE 802.16 Based Vehicular
Networks," IEEE Transaction on vehicular Technology, vol. 56, no. 6 , pp. 3358-3370, 2007

[5] Q. Liu, S. Zhou, and G. B. Giannakis, "Cross-layer scheduling with prescribed QoS guarantees in adaptive wireless networks," IEEE Journal of Selected Areas in Communication, vol. 23, no. 5, pp. 1056 - 1066, 2005.

[6] C. Cicconetti, A. Erta, L. Lenzini, and E. Mingozzi, "Performance Evaluation of the IEEE 802.16 MAC for QoS Support," IEEE Transaction on Mobile Computing, pp. 26-38, vol. 6, no. 1, 2007

[7] X. Bai, A. Shami, and Y. Ye, "Robust QoS control in IEEE 802.16 broadband wireless networks," IEEE Transaction on Mobile Computing, vol. 7, no. 4, pp. 416-429, 2008.

[8] D. Hwang, E. Kwon, and J. Lim, "A Virtual Slot Multiple Access for IEEE 802.15.3 High-Rate Wireless Personal Area Networks," in Proc. of Vehicular Technology Conference (VTC), pp. 1-5, 2006.

[9] M. Wu, S. Makharia, H. Liu, D. Li, and S. Mathur, "IPTV Multicast Over Wireless LAN Using Merged Hybrid ARQ With Staggered Adaptive FEC," IEEE Transactions on Broadcasting, vol. 55, no. 2, pp. 363-374, 2009.

[10] G. Egeland and P. Engelstad, "The Availability and Reliability of Wireless Multi-Hop Networks with Stochastic Link Failures," IEEE Journal on Selected Areas in Communications, vol. 27, no. 7, pp. 1132-1146, Sep. 2009.

[11] Yu- A. Ghosh, D. R. Wolter, J. G. Andrews, and R. Chen, "Broadband wireless access with WiMax/802.16: Current performance benchmarks and future potential," IEEE Communication Magazine, vol. 43, no. 2, pp. $129-136,2005$

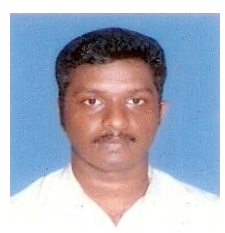

Mr. A. Rajesh received B.Tech and M.Tech degrees in Electronics and Communication Engineering from Pondicherry University in 2005 and 2008 respectively. He has worked at Indian Telephone Industries from 2005 to 2006 and at Tata Consultancy Services from 2008 to 2009. He is a recipient of gold medal at PG level from Pondicherry University and Pondicherry Engineering College. He has been awarded Innovation in Science Pursuit for Inspired Research (INSPIRE) fellowship under assured opportunity for research career (AORC) from the Department of Science and Technology (DST), Ministry of Science and Technology (MST), Government of India. He is a life member of ISTE and IACSIT. He is currently working towards the $\mathrm{PhD}$ degree at the Department of Electronics Engineering, School of Engineering and Technology, Pondicherry Central University, Puducherry. His current research interest is in the areas of WiMAX, WLAN, Heterogeneous Networks, IMS, Power Line Communication and Emerging Wireless Networks

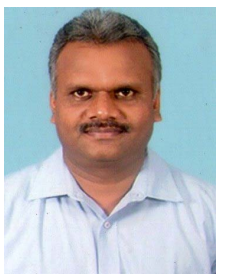

R. Nakkeeran was born in Tamilnadu, India, in 1967. He received B.Sc. degree in Science and B.E degree in Electronics and Communication Engineering from Madras University in 1987 and 1991 respectively and M.E degree in Electronics and Communication Engineering (with diversification in Optical Communication) from Anna University in 1995. He received $\mathrm{PhD}$ degree from Pondicherry University in 2004. Since 1991, he has been working in teaching profession. Presently, he is Associate Professor in the Department of Electronics Engineering, School of Engineering and Technology, Pondicherry Central University. He is life member of IETE, ISTE, OSI and IE (I). Also, he is member of OSA, and SPIE. He has published around 125 papers in National and International Conference Proceedings and Journals. He has co-authored a book, published by PHI. His areas of interest are Optical Communication, Networks, Antennas, Electromagnetic Fields and Wireless Communication. 